Pathologe 2012 · 33:99-102

DOI 10.1007/s00292-012-1569-9

(c) Springer-Verlag 2012

\section{G. Mikuz}

Institut für Pathologie, Medizinische Universität Innsbruck, Österreich

\title{
Die Prostatabiopsie - eine unendliche Geschichte
}

Die Urologen haben das PSA erfunden, die Geißel der Pathologen. Aber diese haben sich mit ASAP fürchterlich gerächt! ${ }^{1}$

Im Zeitraum 1951 bis Januar 2012 findet man in der elektronischen Datenbank PubMed/Medline ${ }^{\circledast}$ unter dem Begriff "Prostatabiopsie“ die unglaubliche Zahl von knapp 43.000 Publikationen. Diese „unbereinigte“ Zahl ist ein sehr grober und oberflächlicher Maßstab, weil viele Publikationen aus den früheren Jahren nur marginal mit der Prostatabiopsie im heutigen Sinn etwas zu tun hatten. Mehr als drei Viertel aller diesbezüglichen Publikationen wurden erst in den letzten 2 Jahrzehnten publiziert. Die Ursache des rasanten Anstiegs der Publikationen in den nachfolgenden Jahren ist die zunächst zaghafte [31], dann aber routinemäßige und sogar systematische serologische Bestimmung des prostataspezifischen Antigens ( $\mathrm{PSA}^{2}$; sog. Männerscreening) für die Entdeckung eines klinisch stummen Prostatakarzinoms [5]. Es soll nicht unerwähnt bleiben, dass die ersten PSA-Untersuchungen an Prostatakarzinomen von Pathologen immunhistochemisch durchgeführt wurden [32] - und dies erst ganze 11 Jahre, nachdem der Immunologe Ablin [1] dieses Gewebsantigen (kein Tumormarker!) isoliert hatte.

Die Pathologen wurden plötzlich mit der Diagnostik eines symptomlosen und unsichtbaren Tumors konfrontiert, der

\footnotetext{
1 Leicht modifiziert nach Dr. Marco Grasso, Chefarzt der Klinik für Urologie am Ospedale San Gerardo dei Tintori in Monza - Universität Mailand Campus Bicocca.

2 Der PSA-Test wurde erst 1994 von der USA Food and Drug Administration zur Anwendung freigegeben.
}

sich nur durch erhöhte Werte von PSA im Blut bemerkbar machte. Die erhöhten Werte sind an und für sich kein sicherer Nachweis für ein bestehendes Karzinom, weil auch bei Entzündungen und mechanischer Reizung (Sport), die Werte erhöht sind. Deswegen versucht man, mit anderen serologischen Parametern (freies PSA, PSA-Dichte, PSA-Verdopplungszeit) eine etwas sicherere serologische Krebsvorhersage zu erreichen [19]. Zurzeit befinden sich einige molekulare Marker als möglicher Ersatz für das PSA-Screening in der Testphase, wobei 2 RNA-Marker, die im Harn bestimmt werden, am aussichtsreichsten erscheinen. Das bisher am häufigsten getestete PCA3 ist eine nichtkodierende RNA, die offensichtlich nur im Prostatagewebe vorkommt. Der bereits kommerziell erhältliche Test (Progensa ${ }^{\circledR}$ PCA3) hat eine 60\%ige Sensitivität. Der Nachweis von PCA3 dient v. a. zur Indikation einer Rebiopsie [26, 27]. Einen höheren positiven prädiktiven Wert (94\%) hat der Nachweis der TMPRSS2:ERG-Genfusion an Prostatazellen im Harn [26, 27], wobei diese Methode allerdings außerordentlich aufwendig ist. Von der Kombination beider Tests wird eine deutlich bessere Treffsicherheit erwartet.

Die Beweisführung für ein erhöhtes serologische PSA traf und trifft aber nur die Pathologen. Nach Einführung des PSA-Screenings vor 20 Jahren standen sie plötzlich nicht nur vor einer riesigen Zahl von Prostatabiopsien (z. B. in den USA >1 Mio./Jahr) sondern auch einer fast gänzlich unbekannten Pathomorphologie gegenüber. Die prostatische intraepitheliale Neoplasie (PIN), die atypische adenomatöse Hyperplasie (AAH), die Basalzellenhyperplasie usw. sind morphologische „Entdeckungen“ dieser zwanzigjährigen Ära.

Abgesehen vom morphologischen Neuland verringerte sich auch die Menge des im Einzelfall zu untersuchenden Gewebes dramatisch. Das Volumen des durch dünnere Nadeln gewonnenen Gewebes entspricht bestenfalls der Hälfte (Gauge 16) oder sogar weniger als einem Drittel (Gauge 18) des Gewebsvolumens einer mit der alten Vim-Silverman-Nadel gewonnenen Biopsie. Mehrfachbiopsien erhöhen zwar die Wahrscheinlichkeit der Entdeckung eines bestehenden Prostatakrebses, verringern aber keinesfalls die Irrtumswahrscheinlichkeit!

Der angesehene amerikanische Urologe Andriole bezeichnete in einem Review [4] die konventionelle Prostatabiopsie als ein Lotteriespiel, bei dem das Karzinom oft verfehlt wird, und falls es entdeckt wird, werden dessen Eigenschaften (Größe, Lokalisation im Organ und Gleason-Score) meist falsch bewertet. Die Diskussion über die notwendige Anzahl von Biopsien für das sichere Aufspüren des Prostatakarzinoms ist unter Urologen noch immer voll im Gange [6, 10]: Minimalisten bescheiden sich mit 10 Biopsien, die Maximalisten verlangen 32 „Sättigungsbiopsien“ (fast eine „Nadelprostatektomie“), zumindest nach einer vorausgegangenen negativen Biopsieserie. Tatsächlich zeigen aber seriöse Untersuchungen, dass man mit 32 Biopsien keine höhere Treffsicherheit erreicht als mit 18 Biopsien [10]. Wir Pathologen sind bei diesem Treiben nur Zaungäste - eine winzige kleindrüsige Proliferation (ASAP: „atypical small acinar proliferation") in einem Biopsiezylinder ist ein 
diagnostisches Problem, das in keiner Beziehung zur Zahl der Biopsien steht.

Im vorliegenden Heft analysieren Helpap u. Oehler [17] die Fragestellungen der Konsiliarbefunde, die sie im Jahr 2008 erhielten, und vergleichen diese mit Befunden aus zwei früheren Jahren $(1995,2003)$. Das Einholen einer Zweitmeinung bei malignitätsverdächtigen Prostataläsionen ist zwischenzeitlich in ganz Europa gängige Praxis, zumal in großen Prostatazentren auch bei zweifelsfreien auswärtigen Diagnosen der Urologe eine Zweitbegutachtung durch den "hauseigenen“ (falls vorhanden) Uropathologen wünscht. Dabei geht es primär um die Interpretation der Befunde, die für die Therapieplanung wichtig sind. Nebenbei kann in diesem Zusammenhang mit Freude festgestellt werden, dass sich die Uropathologie bei der jungen Generation europäischer $\mathrm{Pa}$ thologen offensichtlich großer Beliebtheit erfreut und daher auch ausreichend Pathologen mit entsprechender Expertise zur Verfügung stehen.

Helpap u. Oehler [17] weisen auf die Tatsache hin, dass eine Reihe von diagnostischen Schwierigkeiten oder gar Fehlleistungen nicht durch mangelnde diagnostische Fähigkeiten, sondern durch technische Probleme verursacht werden. Fehler und diagnostische Unsicherheiten sind durch Anwendung standardisierter Einbettungsmethoden [25] und mit guter Schnitt- und Färbequalität größtenteils vermeidbar. Auch die Immunhistochemie ist kein Allheilmittel, welches mittels positiver oder negativer Reaktionen und fröhlichen Farben eine sichere Diagnose verspricht. Eine positive Razemase ( $\alpha$ Methylazyl-CoA-Razemase)-Reaktivität findet sich manchmal auch in harmlosen Läsionen, und es gibt auch p63-positive Karzinome [23]! Die Razemase-Reaktion ist generell eine launische Diva, deren „Erscheinen“ von vielen Faktoren abhängt und sogar in ein und demselben Labor ganz unterschiedliche Ergebnisse liefern kann [20].

Abseits von den nüchternen Zahlen, die die umfangreiche Konsiliartätigkeit der Autoren detailliert belegen, ist für mich der Vergleich der Fragestellungen der Einsender in den verschiedenen Zeitperioden die wichtigste Botschaft dieser Arbeit. In den 1990er Jahren standen offensichtlich die Fragen der Abgrenzungen von $\mathrm{AAH}$ und PIN von Karzinomen und die Unterscheidung zwischen den Gleason-Scores 6 und 7 im Vordergrund. 2008 ist das „Interesse" für AAH und PIN signifikant geringer geworden, dafür stehen ASAP und der Gleason-Score im Vordergrund. AAH und PIN haben offensichtlich ihre „Faszination" verloren und stellen in der täglichen Diagnostik auch kein differenzialdiagnostisches Schreckgespenst mehr dar. Interessanterweise werden Fälle mit Atrophie nicht näher besprochen - die proliferative Form der Atrophie [7] ist nach eigener Erfahrung die häufigste Ursache tragischer Fehldiagnosen.

Man kann Murphy [22] nur beipflichten, wenn er meint, dass es keine wirklich gute Idee ist, unsere diagnostischen Unsicherheiten hinter einem Kürzel (ASAP) $\mathrm{zu}$ verstecken, das wie eine Diagnose klingt. Anständigerweise sollte man einfach mitteilen, dass eine sichere Diagnose nicht möglich ist. Aber, so wie viele andere unkorrekte medizinische Bezeichnungen (wie z. B. die im angelsächsischen Sprachraum gebräuchliche Prostatahypertrophie), ist auch ASAP aus der Uropathologie nicht mehr wegzudenken. Die Diagnose ASAP bedeutet eine fast 50\%ige Wahrscheinlichkeit, dass in der nachfolgenden Rebiopsie ein Karzinom gefunden wird. Dies ist ein wesentlich höher Prozentsatz, als bei der vor 10 Jahren „modernen“ Diagnose HGPIN („High-grade-PIN“) in der Vorbiopsie. Die HGPIN hat selbst nach Einschätzung ihres Erfinders Bostwick [28] deutlich an prädiktiver Bedeutung verloren. Die Ursache für diesen Niedergang liegt nach Meinung der Experten [28] einerseits in den extensiven Biopsietechniken, die schon primär mehr Karzinome zutage fördern und die PIN als Indikator der Notwendigkeit einer Rebiopsie bedeutungslos machen würden. Andererseits sind die von PIN begleiteten Karzinome meist so klein, dass sie unentdeckt blieben oder aber gibt es PIN auch ohne „Begleitkarzinom“.

ASAP ist eine klassische Diagnose, die durch schlechtes oder nicht richtig verarbeitetes Biopsiematerial entsteht. Werden die Schnitte für die Immunhistochemie gleichzeitig mit denen für die HEFärbung (sog. Intervallschnitte) hergestellt, gehen nur $8 \%$ der vorhandenen Kar- zinome verloren. Wird der Paraffinblock nachträglich geschnitten (sog. ,Re-cut") sind es über 50\% [16]! Tatsächlich bestätigen viele Publikationen, dass viele ASAP a priori bereits vom Pathologen nicht erkannte Karzinome sind [17]. Nichtsdestotrotz wird es immer Fälle geben, die trotz perfekter Technik und Zweitmeinung durch ausgewiesene Experten unklar bleiben. Die Urologen wissen, dass in solchen Fällen der Patient rebiopsiert werden muss - „as soon as possible“! Für die Pathologen gibt es ganz einfache Regeln für die Selbstkontrolle der diagnostischen und technischen Qualität von Prostatabiopsien [12]:

- die Durchschnittslänge der Biopsiezylinder (gemessen am Objektträger) sollte $10 \mathrm{~mm}$ betragen,

- eine ASAP-Häufigkeit <3\% ist gut, ASAP $<5 \%$ ist noch annehmbar,

- letztlich darf, nach Kontrolle, die Zahl falsch-negativer Diagnosen 3\% nicht übersteigen.

Die Gleason-Exegeten treffen sich alle paar Jahre, um in den Originalzeichnungen von Gleason [13] weitere Geheimnisse zu entdecken. Beim letzten Treffen 2005 ist das Gleason-System verschärft, aber auch wesentlich vereinfacht worden. Deshalb erscheint es durchaus verwunderlich, dass unter den von Helpap u. Oehler [17] aufgeführten Konsultationsfällen noch Fälle mit Gleason-Score 3 (Grade 1+2), 4 (Grade $2+2$ ) und 5 (Grade $2+3$ ) vorzufinden sind. Die einfachen Regeln lauten: Gleason-Grad 1 gibt es nicht und Grad 2 ist der typische Tumor der transitionalen Zone, dem man praktisch ausschließlich im TUR (transurethrale Resektion)-Material als inzidentales Karzinom begegnet. In der Biopsiediagnostik sollte man die Gleason-Grade 1 und 2 nicht verwenden.

Die wesentliche Vereinfachung betrifft aber die Bewertung der kribrösen (siebähnlichen) Drüsen, die in Gleasons Originalzeichnungen bei Grad 2 (ganz klein), 3 und $4 \mathrm{zu}$ finden sind. Die Gleason-Experten haben mit gutem Grund entschieden, dass, unbeschadet der Morphologie dieser kribrösen Strukturen, ihre Anwesenheit Gleason-Grad 4 bedeutet [13].

Am 9. März 2010 veröffentlichte der PSA-Entdecker Richard Ablin [2] in „The New York Times“ einen Artikel mit dem 
Titel „The great prostate mistake" mit folgendem Statement:

"I never dreamed that my discovery four decades ago would lead to such a profit-driven public health disaster. The medical community must confront reality and stop the inappropriate use of P.S.A. screening. Doing so would save billions of dollars and rescue millions of men from unnecessary, debilitating treatments. "

Ähnlich wie die Väter der Atombombe, die sich von ihrer unheilbringenden Entdeckung nach und nach lossagten, versucht Ablin [2] mit seinem pathetischen Aufruf, eine Entwicklung zu stoppen, die sich mit neuen Testmethoden langsam bereits in die Post-PSA-Screening-Ära zu bewegen beginnt.

Die Reaktionen waren sowohl in den USA $^{3}$ und mehr noch in Europa heftig [15]. Dem Aufruf lag eine Publikation im New England Journal of Medicine zugrunde, in der gezeigt wurde, dass das PSAMassenscreening in den USA keine Verringerung der Mortalität bei Prostatakrebs bewirkt hatte [3]. Ganz im Gegensatz da$\mathrm{zu}$ zeigen europäische Studien eine langsame, aber deutliche Abnahme der Prostatakrebsmortalität eben durch das PSAScreening [33]. Tatsächlich erkennt man in der Zwischenzeit auch in den USA an, dass beide Studien nicht vergleichbar sind [21] und dass im Gegensatz zur zitierten US-amerikanischen Studie die europäischen Studien methodisch einwandfreier durchgeführt wurden. Nichtsdestotrotz stellt sich auf beiden Seiten des Atlantiks die berechtigte Frage, ob die Prostatektomie tatsächlich die einzige Therapieoption für die potenziell heilbaren Prostatakarzinome sei. Es ist nämlich hinlänglich bekannt, dass nach dem 50. Lebensjahr, zwar 50\% der Männer ein latentes Karzinom haben, aber nur bei $10 \%$ wird es klinisch manifest $-d$. h. die Mehrzahl wird mit, aber nicht an Prostatakrebs sterben [9]. Deswegen heißt das magische Wort nun „active surveillance“, $d$. h. dass unter bestimmten Voraussetzungen ein Patient

\footnotetext{
3 In einem Leserbrief erinnert der Urologe John C. McHugh M.D. daran, dass der Vater des Immunologen Ablin elendig an einem metastasierenden Prostatakrebs verstarb und dass dieser Tod durch zeitgerechtes PSA-Screening vermeidbar gewesen wäre.
}

mit Prostatakrebs nur aktiv beobachtet wird $[14,30]$. „Aktiv“ bedeutet aber auch, dass Prostatabiopsien gegebenenfalls vermehrt entnommen werden müssen.

Die wichtige Voraussetzung für die aktive Überwachung wäre die Feststellung, ob es sich um einen sog. ,insignifikanten Prostatakrebs“ handelt. Die klar definierten Kriterien [11] für den insignifikanten Prostatakrebs sehen vor, dass das Karzinom in nicht mehr als zwei Biopsiezylindern vorhanden sein darf. Es wird nunmehr von mehreren Prostatazentren versucht, diese grobe Quantifizierung durch eine präzisere zu ersetzen $[18,24]$. Es ist anzunehmen, dass die Standardisierung der Karzinommessmethoden am Biopsiezylinder eines der Kernthemen kommender Konsensuskonferenzen sein wird, um diesbezüglich verbindliche Leitlinien $\mathrm{zu}$ klinischen Normogrammen für die Therapieplanung zu erarbeiten [29].

Ob nun Prostatektomie, Surveillance oder andere therapeutische Optionen es ist in nächster Zukunft nicht zu erwarten, dass ohne Prostatabiopsien klinische Entscheidungen getroffen werden. Der Einsatz neuer morphologischer Parameter und vielleicht sogar eine neue Deutung des Gleason-Gradings [8] werden wieder neue Diskussionen entfachen. Wie eingangs gesagt... eine unendliche Geschichte!

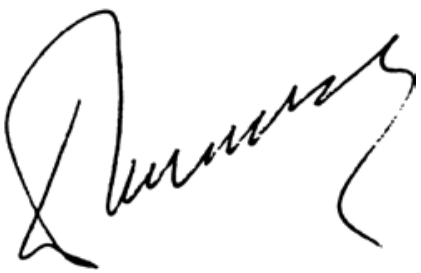

Prof. Dr. G. Mikuz

\section{Korrespondenzadresse}

Em. o. Prof. Dr. G. Mikuz

Institut für Pathologie, Medizinische Universität Innsbruck

Müllerstr. 44, 6020 Innsbruck

Österreich

Gregor.Mikuz@i-med.ac.at

Interessenkonflikt. Der korrespondierende Autor gibt an, dass kein Interessenkonflikt besteht.

\section{Literatur}

1. Ablin RJ, Soanes WA, Bronson P et al (1970) Precipitating antigenes of the normal human prostate. J Reprod Fert 22:573-574

2. Ablin RJ (2010) The great prostate mistake. The New York Times, 9. März, S 27

3. Andriole GL, Crawford DE, Grubb RL III et al (2009) Mortality results from a randomized prostate-cancer screening trial. N Engl J Med 360:1310-1319

4. Andriole GL (2009) Pathology: the lottery of conventional prostate biopsy. Nat Rev Urol 6:188-189

5. Bartsch G, Horninger W, Klocker H et al (2001) Prostate cancer mortality after introduction of prostate-specific antigen mass screening in the Federal State of Tyrol, Austria.Urology 58:417-424

6. Chun FK, Epstein JI, Ficarra V et al (2010) Optimizing performance and interpretation of prostate biopsy: a critical analysis of the literature. Eur Urol 58:851-864

7. De Marzo AM, Platz EA, Epstein Jl et al (2006) A working group classification of focal prostate atrophy lesions. Am J Surg Pathol 30:1281-1291

8. Delahunt B, Miller RJ, Srigley JR et al (2012) Gleason grading: past, present and future. Histopathology 260:75-86

9. Delongchamps NB, Singh A, Haas GP (2006) The role of prevalence in the diagnosis of prostate cancer. Cancer Control 13:158-168

10. Delongchamps NB, de la Roza G, Jones R et al (2009) Saturation biopsies on autopsied prostates for detecting and characterizing prostate cancer. BJU Int 103:49-54

11. Epstein Jl, Walsh PC, Carmichael M et al (1994) Pathologic and clinical findings to predict tumor extent of nonpalpable (stage T1c) prostate cancer. JAMA 271:368-374

12. Epstein Jl (1999) How should atypical prostate needle biopsies be reported? Controversies regarding the term „ASAP”. Hum Pathol 30:1401-1402

13. Epstein Jl, Allsbrook WC Jr, Amin MB, Egevad LL; ISUP Grading Committee (2005) The 2005 International Society of Urological Pathology (ISUP) consensus conference on Gleason grading of prostatic carcinoma. Am J Surg Pathol 29:1228-1242

14. Gorin MA, Eldefrawy A, Ekwenna O et al (2011) Active surveillance for low-risk prostate cancer: knowledge, acceptance and practice among urologists. Prostate Cancer Prostatic Dis [Epub ahead of print]

15. Graefen M, Gschwend J, Hammerer et al. Für den Vorstand der AUO (2010) Stellungnahme zum Artikel „Der große Prostata-Irrtum" von Richard Ablin; www.lifepr.de/pressemeldungen/deutsche-krebsgesellschaft

16. Hameed O, Humphrey PA. (2009) Immunohistochemical evaluation of prostate needle biopsies using saved interval sections vs new recut sections from the block: a prospective comparison. Am J Clin Pathol131:683-687

17. Helpap B, Oehler U (2012) Bedeutung der Zweitmeinung bei Prostatabiopsien. Pathologe 33 [Epub ahead of print]

18. Humphrey PA (2009) Tumor amount in prostatic tissues in relation to patient outcome and management. Am J Clin Pathol 131:7-10

19. Loeb S, Catalona WJ (2007) Prostate-specific antigen in clinical practice. Cancer Lett 249:30-39

20. Magi-Galluzzi C, Luo J, Isaacs WB et al (2003) Alpha-methylacyl-CoA racemase: a variably sensitive immunohistochemical marker for the diagnosis of small prostate cancer foci on needle biopsy. Am J Surg Pathol 27:1128-1133 
21. McNaughton-Collins MF, Barry MJ (2011) One man at a time - resolving the PSA controversy. N Engl J Med 365:1951-1953

22. Murphy WM (1999) ASAP is a bad idea. Atypical small acinar proliferation. Hum Pathol 30:601

23. Osunkoya AO, Hansel DE, Sun X et al (2008) Aberrant diffuse expression of p63 in adenocarcinoma of the prostate on needle biopsy and radical prostatectomy: report of 21 cases. Am J Surg Pathol 32:461-467

24. Quintal MM, Meirelles LR, Freitas LL et al (2011) Various morphometric measurements of cancer extent on needle prostatic biopsies: which is predictive of pathologic stage and biochemical recurrence following radical prostatectomy? Int Urol Nephrol 43:697-705

25. Rogatsch $\mathrm{H}$, Mairinger T, Horninger W et al (2000) Optimized preembedding method improves the histologic yield of prostatic core needle biopsies. Prostate 42:124-129

26. Roobol MJ, Haese A, Bjartell A. (2011) Tumor markers in prostate cancer III: biomarkers in urine. Acta Oncol 50 (Suppl 1):85-89

27. Salagierski M, Schalken JA (2012) Molecular diagnosis of prostate cancer: PCA3 and TMPRSS2:ERG gene fusion. J Urol [Epub ahead of print]

28. Schlesinger C, Bostwick DG, Iczkowski KA (2005) High-grade prostatic intraepithelial neoplasia and atypical small acinar proliferation: predictive value for cancer in current practice. Am J Surg Pathol 29:1201-1207

29. Shariat SF, Karakiewicz PI, Suardi N (2008) Comparison of normograms with other methods for predicting outcomes in prostate cancer: a critical analysis of the literature. Clin Cancer Res 14:44004407

30. Slomski A (2012) Expert panel advocates surveillance for men with low-risk prostate cancer. JAMA 307:133

31. Soula G (1985) Cancer de la prostate: les marquers autres que la phosphatase acide prostatique. Bull Cancer 72:527-530

32. Stein BS, Vangore S, Petersen RO, Kendall AR (1982) Immunoperoxidase localization of prostatespecific antigen. Am J Surg Pathol 6:553-557

33. Verdecchia A, Guzzinati S, Francisci S, De Angelis R; EUROCARE Working Group (2009) Survival trends in European cancer patients diagnosed from 1988 to 1999. Eur J Cancer 45:1042-1066

\section{Allgemeines}

Angabe eines Interessenkonflikts im Manuskript (weitere Informationen auf der Seite „Erklärung zum Interessenkonflikt")

Manuskript immer als Datei schicken (.doc oder .rtf, keine PDF-Dateien)

Gesamtumfang: 12 Manuskriptseiten (Times New Roman, 12 pt, 2-zeilig) inkl. Literatur, Tabellen und Abbildungslegenden (entspricht ca. 25000 Zeichen, inkl. Leerzeichen)

$\checkmark$ Für die Angaben von Maßeinheiten bitte das SI-System verwenden

$\square$ Abkürzungen im Text erläutern, ggf. Abkürzungsverzeichnis erstellen

\section{Manuskriptaufbau}

Komplette Anschrift des Korrespondenzautors mit Tel.-Nr., Fax, E-Mail sowie Portraitfoto

Kurzer, prägnanter Beitragstitel (ca. 50 Zeichen), ggf. erläuternder Untertitel

Deutsche Zusammenfassung (max. 1200 Zeichen, inkl. Leerzeichen). Bei Überschreitung behält sich der Verlag Kürzungen vor

5 deutsche Schlüsselwörter Englischer Titel

Englisches Abstract (max. 1200 Zeichen, inkl. Leerzeichen). Abstract und Zusammenfassung sollen inhaltlich identisch sein; möglichst einheitlich British English oder American English verwenden

5 englische Keywords zur besseren Auffindbarkeit in Datenbanken unter Verwendung der "Medical Subject Heading (MeSH)"-Liste des Index Medicus (http://www.nIm. nih.gov/mesh/authors.html)

$\square$ Kurze Hinführung zum Thema (max.600 Zeichen)

$\square$ Prägnante und möglichst kurze Zwischenüberschriften (max. 50 Zeichen). 4 Hierachien sind möglich

Fazit für die Praxis (max. 1000 Zeichen, inkl. Leerzeichen)

\section{Literaturverzeichnis}

Maximal 30 Literaturstellen in alphabetischer Reihenfolge und durchnummeriert

$\square$ Zitatnummern im Text in eckige Klammern setzen

$\square$ Bei Benutzung von „Endnote“: bitte Literaturstyling „Springer Fachzeitschrift MedizinPsychologie" verwenden

Zeitschriftentitel nach Index Medicus abkürzen

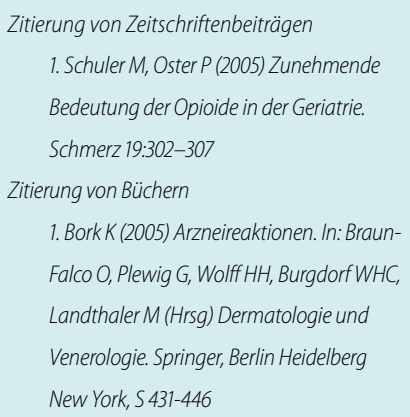

\section{Abbildungen}

Max. 8 Abbildungen mit kurzen Legenden sind möglich

$\square$ Auf alle Abbildungen im Text verweisen

$\square$ Abbildungen entsprechend der Reihenfolge im Text durchnummerieren

Deutsche Abbildungsbeschriftung

Formate: tif, jpg, ppt, eps

Auflösung mind. $300 \mathrm{dpi}$

Unbefristete Abdruckgenehmigung (print/online/offline) für Abbildungen aus Fremdpublikationen

$\square$ Einverständniserklärung identifizierbarer Personen

\section{Videomaterial}

Formate: $640 * 480$ (4:3) Pixel, Container MOV, Video-Codec MPEG-4, Audio-Codec ACC, 96kbps, 25fps

\section{Tabellen}

Aussagekräftige Tabellen mit kurzen Tabellenüberschriften sind erwünscht

Auf alle Tabellen im Text verweisen

Tabellen entsprechend der Reihenfolge im Text durchnummerieren 